\title{
BMJ Open Bridge study protocol: an international, observational cohort study on the transition of healthcare for adolescents with chronic conditions
}

\author{
Silja Kosola (10 , ,,2 Evelyn Culnane, ${ }^{3}$ Hayley Loftus, ${ }^{3,4}$ Anna Tornivuori, ${ }^{5}$ \\ Mira Kallio, ${ }^{1,2,6}$ Michelle Telfer, ${ }^{7,8}$ Päivi J Miettinen, ${ }^{1,2}$ Kaija-Leena Kolho, , ,2,9 \\ Kristiina Aalto, ${ }^{1,2}$ Taneli Raivio, ${ }^{1,2,10}$ Susan Sawyer ${ }^{4,7,8,11}$
}

To cite: Kosola S, Culnane E, Loftus $\mathrm{H}$, et al. Bridge study protocol: an international, observational cohort study on the transition of healthcare for adolescents with chronic conditions. BMJ Open 2021;11:e048340. doi:10.1136/ bmjopen-2020-048340

- Prepublication history for this paper is available online. To view these files, please visit the journal online (http://dx.doi org/10.1136/bmjopen-2020048340).

Received 22 December 2020 Accepted 18 May 2021

Check for updates

(c) Author(s) (or their employer(s)) 2021. Re-use permitted under CC BY-NC. No commercial re-use. See rights and permissions. Published by BMJ.

For numbered affiliations see end of article.

Correspondence to

Dr Silja Kosola;

silja.kosola@helsinki.fi

\section{ABSTRACT}

Introduction More than $10 \%$ of adolescents live with a chronic disease or disability that requires regular medical follow-up as they mature into adulthood. During the first 2 years after adolescents with chronic conditions are transferred to adult hospitals, non-adherence rates approach $70 \%$ and emergency visits and hospitalisation rates significantly increase. The purpose of the Bridge study is to prospectively examine associations of transition readiness and care experiences with transition success: young patients' health, health-related quality of life (HRQoL) and adherence to medical appointments as well as costs of care. In addition, we will track patients' growing independence and educational and employment pathways during the transition process.

Methods and analysis Bridge is an international, prospective, observational cohort study. Study participants are adolescents with a chronic health condition or disability and their parents/guardians who attended the New Children's Hospital in Helsinki, Finland, or the Royal Children's Hospital (RCH) in Melbourne, Australia. Baseline assessment took place approximately 6 months prior to the transfer of care and follow-up data will be collected 1 year and 2 years after the transfer of care. Data will be collected from patients' hospital records and from questionnaires completed by the patient and their parent/guardian at each time point. The primary outcomes of this study are adherence to medical appointments, clinical health status and HRQOL and costs of care. Secondary outcome measures are educational and employment outcomes. Ethics and dissemination The Ethics Committee for Women's and Children's Health and Psychiatry at the Helsinki University Hospital (HUS/1547/2017) and the RCH Human Research Ethics Committee (38035) have approved the Bridge study protocol. Results will be published in international peer-reviewed journals and summaries will be provided to the funders of the study as well as patients and their parents/guardians.

Trial registration number NCT04631965.

\section{INTRODUCTION}

One measure of the success of contemporary paediatric practice is the extent to which children with severe chronic health conditions
Strengths and limitations of this study

- Bridge is the first international study to prospectively study the transition of adolescents with a chronic condition and/or disability that focuses immediately before transfer and up to 2 years after they have transferred from paediatric to adult health services.

- The comprehensive outcome measures will provide unique information about physical and emotional health and well-being as well as the social and educational challenges the adolescents face at this time.

- Understanding commonalities and differences in transition pathways and outcomes in two countries with different cultures and health and social systems will contribute to identifying groups of patients who might benefit from more individualised transition planning.

- Loss of follow-up and variable access to health data may prove challenging, and understanding causation will also be limited as differences between countries may reflect cultural differences as well as different health systems.

- The COVID-19 pandemic may affect many factors including delayed transfer to adult services, delayed follow-up by adult services, as well as participant hesitancy to attend adult services and engage in follow-up assessments given the greater impact of the pandemic on adult health services.

and disabilities that were previously fatal in childhood, such as congenital heart defects and spina bifida, are now expected to survive into adulthood. ${ }^{1}$ Concurrently, the incidence of various conditions, such as diabetes and inflammatory bowel diseases, has increased. $^{2-4}$ These changing health profiles have led to increases in the number of adolescents whose specialist medical care must be transferred from paediatric to adult settings, which has been estimated to affect at least 1 in 10 adolescents. ${ }^{5}$ 
The concept of transition, the planned process of transferring healthcare from specialist paediatric to adult services, was introduced almost 30 years ago. ${ }^{6}$ Since then, recommendations for how to support the process of transition have been developed, commonly in reference to specific diagnoses but also within generic national guidelines. ${ }^{7-13}$ Commonalities within these recommendations include the importance of transition planning starting early, support for adolescents' self-efficacy and self-management and the need to consider individual circumstances.

Adolescence, and the transitions of care that accompany it, can be a challenging time for young people, their families and health professionals. Adolescents leaving their familiar child health professionals can find involvement with new professionals difficult ${ }^{14} 15$ and parents can struggle with expectations that they are less directly engaged in adult services. Professionals who are used to treating adult patients may lack knowledge about childhood onset conditions, resources and training required to work with adolescents and young adults. ${ }^{16-19}$ During the first years after adolescents with chronic health conditions are transferred to adult hospitals, adherence to treatment and follow-up regimes decreases and emergency visits and hospitalisation rates increase significantly, ${ }^{20}$ suggesting the value of efforts to promote continuity of both primary and specialty healthcare at this time. ${ }^{21}$

Despite the rising number of publications about transition to adult healthcare, studies are typically small, single-centre studies using cross-sectional or retrospective methods. ${ }^{22}{ }^{23}$ Most studies are of single disorders, most commonly type 1 diabetes. ${ }^{24}$ For example, in a recent systematic review of 37 systematic reviews, only 6 studies included patients from different diagnostic groups. ${ }^{25}$ The transition literature has mostly focused on patients' and paediatric providers' concerns prior to the transfer of care, with less data available about outcomes after the transfer of care. ${ }^{25}{ }^{26}$ Indeed, the brief duration of follow-up within prospective studies commonly precludes assessment of meaningful change in health outcomes $(14,15) .{ }^{1415}$ Anxiety is one of the most frequently identified barriers to successful transition, but its significance to transition outcomes remains unknown. ${ }^{26}$

Cultural differences are also often overlooked in the literature. This has implications for the age at which transfer to adult services occurs, due to variation in the upper age limit of paediatric care between countries, ${ }^{27}$ and even greater differences in the age at which young people move away from their childhood home (which reduces opportunities for parent supervision). For example, in Finland, only $20 \%$ of young people aged 20-24 years still live with their parents, in comparison to $43 \%$ of their Australian peers. ${ }^{28}{ }^{29}$ Countries also differ by the extent of social protections available to support health transitions including subsidised healthcare and health insurance, access to education and employment for people with chronic health conditions and disabilities, and access to supported accommodation. ${ }^{30}$
Poor adherence with medication and medical appointments can result in increased use of emergency departments and more hospital admissions for preventable complications, with commensurately greater costs to the healthcare system than routine care. ${ }^{22}$ Poor health may also affect educational outcomes. Even in Finland, where both education and healthcare provision are publicly funded, adolescents with chronic health conditions are more likely to drop out of formal education than their peers. ${ }^{31}$ Beyond disease control, other life transitions around education and employment typically occur at a similar time as the health transition but with little understanding of how these different transitions relate to each other. $^{32}$

Pleasingly, there is international consensus about indicators and outcomes of a successful healthcare transition. These include health outcomes such as stable disease control, subjective outcomes such as quality of life and patient and family satisfaction with transfer of care and health service outcomes, such as the timing of the first consultation with the adult service being within 3-6 months after transfer and regular attendance at medical appointments. ${ }^{33-35}$

\section{Study aims and hypotheses}

The three aims of this prospective study are to measure;

1. Changes in health outcomes including health-related quality of life (HRQoL),

2. Healthcare experiences and their association with adherence to care, and

3. Changes in anxiety related to transition among a cohort of Finnish and Australian young people with chronic health conditions and/or disabilities over the course of their transition from paediatric to adult services.

Our secondary aims are to assess how well the study centres adhere to international transition guidelines and their key elements, and to estimate the costs of transitional care. We also aim to assess adolescents' participation in education and employment across these years.

Our primary hypothesis is that transition readiness and general self-efficacy will be stronger predictors of adherence to follow-up appointments, fewer emergency visits and continued education than chronological age. Our secondary hypothesis is that positive experiences of care will be associated with lower levels of anxiety across the healthcare transition and that positive care experiences and low anxiety will predict better health outcomes, better quality of life and lower costs of care.

\section{METHODS AND ANALYSIS}

\section{Setting}

Bridge is an international, prospective cohort study that will be conducted at two study centres: the New Children's Hospital (NCH), Helsinki, Finland, and the Royal Children's Hospital (RCH), Melbourne, Australia. In Finland, most participants will transfer to the Helsinki University 


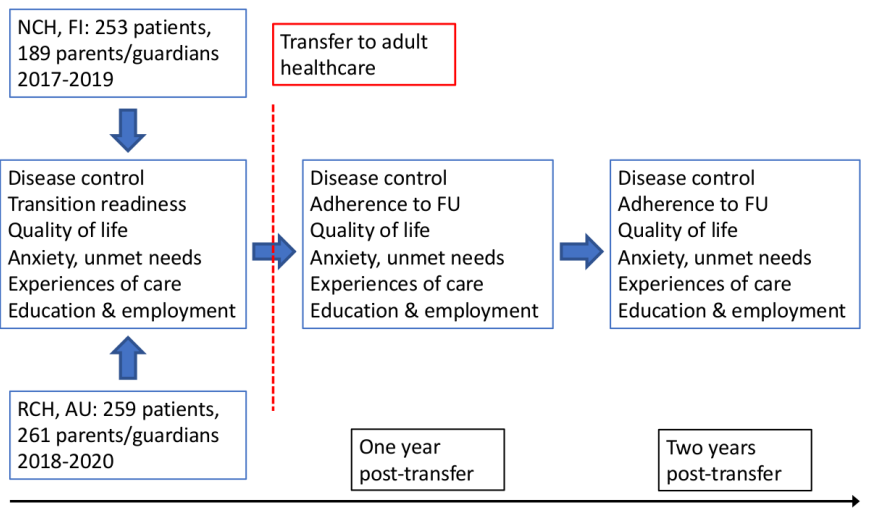

Figure 1 Flowchart depicting the overall Bridge study design. AU, Australia; FI, Finland; FU, follow-up; NCH, New Children's Hospital; RCH, Royal Children's Hospital. Please note: after the transfer of care, parents/guardians in Finland will only be able to participate, if their child is still under the age of 18 years.

Hospital but some will also transfer to other hospitals and healthcare services in Finland. In Melbourne, most participants will transfer to hospitals in metropolitan Melbourne but some will transfer to health services in regional and rural Victoria.

The general study design is described in figure 1. Some similarities and differences between the two study centres relevant to young people with chronic conditions are summarised in table 1 .

At both study centres, transition is viewed as a systematic process and all subspecialties have access to common resources, such as checklists to prepare patients for the transfer of care.

\section{Eligibility criteria}

At recruitment, inclusion criteria for adolescents were that they: were aged 15-24 years; had a chronic health condition and/or disability that was diagnosed at least 6 months earlier; require regular follow-up at adult health services; and are expected to transfer from paediatric to adult care facilities within 6-12 months after recruitment. They also needed to have the linguistic and cognitive capacities to independently communicate in one of the study languages (Finnish, Swedish or English).

\section{Sample size and power calculations}

To ensure sufficient sample size for all outcome measures, we conducted two power calculations. First, we wished to detect a difference in clinic attendance from $80 \%$ to $90 \%$ between groups of low and high transition readiness. With a power of $90 \%$ and $\alpha=0.05$ (two-sided), 85 patients will be needed in each group. Assuming a dropout rate of $20 \%$, 213 patients will be required. Second, we wished to detect at least a $5 \%$ change in HRQoL over time (from 0.85 to 0.90 or vice versa as measured by the $16 \mathrm{D}$ score). With a power of $90 \%$ and $\alpha=0.05$ (two-sided), we will need 190 patients in each group, which totals to 475 once the same dropout rate of $20 \%$ is factored in. We also set a minimum target of 250 patients at each study site. We conducted one more confirmatory power analysis using the General Linear Mixed Model Power and Sample Size tool. This analysis confirmed that 480 patients would be sufficient to detect changes in repeated measures of HRQoL also using the Paediatric Quality of Life Inventory (PedsQL).

\section{Patient recruitment}

At NCH, healthcare professionals in different subspecialty clinics identified adolescents who were expected to transfer to adult services within the next 6 months. Between September 2017 and August 2019, a research nurse uninvolved in patient care met 306 consecutive, eligible adolescents and their 281 parents/guardians face-to-face when they attended a routine outpatient appointment. The nurse provided patients with both verbal and written information about the Bridge study. A parent/guardian of those younger than 18 years was also invited to participate. Altogether 279 adolescents and 214 parents/guardians (91\% and 76\%, respectively) provided written informed consent. After a maximum of

Table 1 Similarities and differences of the two study sites

\section{New Children's Hospital, Royal Children's Finland Hospital, Australia}

Similarities

Publicly funded healthcare system

Paediatric care for severe chronic conditions mainly hospital-based

High participation in secondary education until age 18

Differences

General upper age of paediatrics, years

Transition support and transfer coordination

Role of primary care in transition

Living with parents at age 20-24, \%

Tertiary education (university, college) $x$

$x-x$

$x+\frac{x}{x}$

$x \quad x$

16 19

Varies between paediatric Hospital-wide support subspecialties service available

Significant especially in Variable involvement diabetes

20 43

No tuition fees for citizens Fees for citizens 
two reminders, 253 adolescents and 189 parents/guardians $(83 \%$ and $67 \%$ of those approached, respectively) completed the baseline survey either via Webropol, a secure online data capture system, or by pen-and-paper. Patients received a movie ticket (value $10 €$ ) for each survey; parents received no reimbursement.

At the RCH, between October 2018 and August 2020 staff from the Transition Support Service invited eligible adolescents to participate in the study when they attended routine transition appointments within 12 months prior to their expected transfer of care. The study coordinator also identified and invited eligible adolescents who did not have an upcoming transition appointment to participate in the study by phone or email. A total of 367 adolescents and 380 parents/guardians were invited, of whom 259 adolescents and 261 parents/guardians provided written informed consent (71\% and $69 \%$, respectively). Participants completed the baseline survey either by pen-and-paper or via REDCap, a secure online data capture system. A parallel approach to recruitment occurred with a parent of eligible participants. Patients received a gift voucher (value \$A10) for each survey; parents received no reimbursement.

\section{Baseline measures, exposures and confounders}

Transition readiness and self-efficacy

The Am I ON TRAC for Adult Care (ON TRAC) questionnaire was used to measure transition readiness. ${ }^{36}$ ON TRAC includes a knowledge scale of 14 items and a behaviour index of 9 items. The knowledge scale scores range from 14 to 56 (and 11 to 44, if adolescents indicate they are not taking any medications). The behaviour index scores range from 9 to 45 (8 to 40 , if adolescents are not using medications). High transition readiness is defined as adolescents responding 'agree or strongly agree' to at least 8 of the 9 items $(7 / 8$, if patient is not using medications) of the behaviour index. Nine additional items were chosen from other generic transition readiness questionnaires (eg, the Transition Readiness Assessment Questionnaire) to provide more detail on adolescent patients' self-efficacy, such as being able to book their own clinic appointments, and managing daily activities such as preparing meals.

\section{Health status}

Adolescents report how much their condition has impacted them during the past week using the Visual Analogue Scale (VAS) (where 1 indicates 'very much' and 7 indicates 'very little') ${ }^{3738}$ Clinical condition-specific markers and treatment details will be obtained from individual patient records for the last year prior to the transfer of care. Participants will be grouped into one of three categories, in the context of the specific disorder: (1) good disease control and/or adherence, (2) some evidence of concern and (3) poor disease control and/or adherence or more severe disease/condition, as described in table $2 .^{39} 40$

HRQoL

Adolescents will complete two measures of HRQoL at each time point. The $16 \mathrm{D}$ is a validated, generic scale

Table 2 Guidelines for grouping patients into three categories according to disease control and/or adherence

\begin{tabular}{|c|c|c|c|}
\hline Diagnosis group & Good control and/or adherence & Some evidence of concern & $\begin{array}{l}\text { Poor control and/or adherence } \\
\text { or more severe condition }\end{array}$ \\
\hline Diabetes & $\begin{array}{l}\text { Mean of } \mathrm{HbA} 1 \mathrm{c} \text { values } \leq 53 \mathrm{mmol} / \\
\text { mol }\end{array}$ & $\begin{array}{l}\text { Mean of } \mathrm{HbA} 1 \mathrm{c} \text { values } 54- \\
69 \mathrm{mmol} / \mathrm{mol}\end{array}$ & $\begin{array}{l}\text { Mean of HbA1c values } \\
\geq 70 \mathrm{mmol} / \mathrm{mol}\end{array}$ \\
\hline Gastroenterology & $\begin{array}{l}\text { VAS } 1-2 ; \text { at least } 80 \% \text { of faecal } \\
\text { calprotectin results }<100 \mu \mathrm{g} / \mathrm{g} \text { and } \\
\text { always }<300 \mu \mathrm{g} / \mathrm{g} \text {; medication } \\
\text { unchanged or reduced; no inpatient } \\
\text { care }\end{array}$ & $\begin{array}{l}\text { VAS } 3-5 ;<80 \% \text { of faecal } \\
\text { calprotectin results within } \\
\text { target range or exceeds } \\
300 \mu \mathrm{g} / \mathrm{g} \text { even once; no } \\
\text { significant medication } \\
\text { changes; no inpatient care }\end{array}$ & $\begin{array}{l}\text { Any one of the following: VAS } \\
6-7 ; \text { changes in medication, } \\
\text { need for oral corticosteroids and/ } \\
\text { or commencement of biological } \\
\text { medication; episode of inpatient } \\
\text { care }\end{array}$ \\
\hline $\begin{array}{l}\text { Rheumatology, } \\
\text { arthritides }\end{array}$ & $\begin{array}{l}\text { Oligoarthritis: JADAS } 10 \text { or } \\
\text { cJADAS } 10 \leq 0.5 \text {; polyarthritis: } \\
\text { JADAS } 10 \text { or } \text { CJADAS } 10 \leq 0.7\end{array}$ & $\begin{array}{l}\text { Oligoarthritis: JADAS10 } \\
\text { or cJADAS10 0.6-2.8; } \\
\text { polyarthritis: JADAS10 or } \\
\text { cJADAS10 0.8-4 }\end{array}$ & $\begin{array}{l}\text { Oligoarthritis: JADAS10 or } \\
\text { cJADAS10 >2.8; polyarthritis: } \\
\text { JADAS10 or CJADAS10 >4 }\end{array}$ \\
\hline
\end{tabular}

Patients with rare conditions (eg, congenital heart defects, solid organ transplants or connective tissue diseases) will be categorised according to symptoms, clinical and laboratory findings and changes in medication.

cJADAS, clinical Juvenile Arthiritis Disease Activity Score; HbA1c, glycated haemoglobin; JADAS10, 10-joint Juvenile Arthritis Disease Activity Score; ORT, outpatient rehabilitation therapy (may include occupational, physical and/or speech therapy); RAH, rehabilitation at home; VAS, Visual Analogue Scale. 
that measures HRQoL in 16 dimensions: mobility, vision, hearing, breathing, sleeping, eating, speech, excretion, discomfort and symptoms, depression, distress, mental function, vitality, appearance, school and hobbies and friends. ${ }^{41}$ Each dimension has five response options. The $16 \mathrm{D}$ yields a single index (range $0-1$ ) that can be used to assess quality adjusted life years and cost-effectiveness. A higher index implies better HRQoL.

The PedsQL is a validated, generic measure. ${ }^{42}$ It consists of 23 items within four domains: physical, emotional, social and school. Items are scored on a 5-point Likert scale (total range 0-100). Inclusion of this second measure is primarily to facilitate international comparisons due to its frequent use.

\section{Anxiety}

The Spielberger State-Trait Anxiety Inventory (STAI) is a self-report instrument of 40 items, scored on a 4-point Likert scale. ${ }^{43}$ Adolescents will complete the six-item short form ${ }^{44}$ at each time point. The introductory statement was modified to indicate how adolescents and parents/guardians feel about managing the health conditions and the transfer of care to adult services. In Australia, non-specific psychosocial distress is also measured using the six-item Kessler 6 (K6) scale. ${ }^{45}$ The K6 yields scores between 6-30, with lower scores indicating higher psychosocial distress.

\section{Experiences of care}

Participants will evaluate their experiences of care before and after transfer using the Adolescent Friendly Hospital Survey which was developed and validated at the RCH. ${ }^{46}$ This survey has 13 indicators, 8 of which are not included within the ON TRAC questionnaire.

\section{Risk-taking behaviour}

Adolescents will complete a validated 10-item questionnaire that explores use of tobacco and other nicotine products, alcohol and drug use and any risky situations that they have encountered while using substances. ${ }^{47}$

\section{Worries and unmet needs}

Adolescents' worries are assessed in a framework based on the HEADSS assessment. ${ }^{48}$ Participants are asked whether they have any worries regarding: home or family life, education or employment, healthy lifestyle (diet, exercise and sleep), friends and activities, emotional wellbeing or mental health, accidents and injuries, substance use (smoking, alcohol, cannabis and other drugs), sexual health and contraception, fertility or abuse. They are also asked about any concerns around confidentiality of healthcare and self-management. For any topic that adolescents are worried about, a further question inquires whether this topic has been discussed at the $\mathrm{NCH}$ or $\mathrm{RCH}$, respectively.

\section{Outcome measures}

Changes in self-efficacy, health status and HRQoL

These will be measured in the 1-year and 2-year follow-up questionnaires using the same tools as at baseline.

\section{Adherence to care}

Adherence to medications and follow-up appointments will be measured 1 and 2 years after the transfer of care by self-report. We will also enquire about common reasons for missing medications and appointments. In Finland, data linkage will be used to acquire data on missed follow-up appointments and emergency admissions due to avoidable causes from hospital administrative data.

\section{Costs of care}

We will use hospital administrative data to estimate the cost of care for each patient during the transition process (1 year before the transfer of care to 2 years after the transfer of care). We will consider costs of actualised care as well as potential costs of more frequent follow-up visits and potential savings of avoidable hospital admissions. ${ }^{49}$

\section{Educational and employment outcomes}

At each time point, we ask adolescents about their educational and employment status and their level of satisfaction about this. Reasons for dissatisfaction will also be sought.

\section{Parent/guardian data \\ Transition readiness}

Parents/guardians will evaluate their child's transition readiness and self-efficacy using ON TRAC, which provides an opportunity for comparison with adolescents' responses.

\section{Health status}

Parents/guardians report how much their child's condition has impacted their child during the past week using the VAS (where 1 indicates 'very much' and 7 indicates 'very little'), similar to adolescents.

\section{Anxiety}

Parents/guardians rate their own level of anxiety in relation to the transfer of their child's healthcare using the STAI six-item short form, similar to adolescents. In Australia, non-specific psychosocial distress among parents/guardians will also be measured using the K6.

\section{Experiences of care}

Parents/guardians will evaluate the experiences of care from their own perspective using the Adolescent Friendly Hospital Survey.

\section{Worries and unmet needs}

Parents'/guardians' worries concerning their child are assessed in a framework based on the HEADSS assessment, similar to adolescents. For any topic that parents/ guardians are worried about, a further question inquires whether this topic has been discussed at the NCH or RCH.

\section{Analysis plan}

We will adhere to the Strengthening the Reporting of Observational Studies in Epidemiology guidelines in reporting quantitative findings. ${ }^{50}$ We will report demographic and descriptive data as numbers and percentages 
for categorical variables and mean values with SD (or in case of skewed distribution, medians with IQRs) for continuous variables.

Since disease activity, HRQoL, experiences of care, transition readiness and anxiety may influence each other, we will conduct cross-sectional analyses to evaluate these interactions. Age, sex and education status will be accounted for as possible confounders, and data will be clustered according to the country of residence, when appropriate.

ORs will be calculated to estimate the predictive value of key transition elements (eg, ON TRAC scores) on clinic attendance, emergency visits and avoidable hospitalisations. Multivariable regression analyses will be conducted to account for confounders in cases of continuous measures (eg, HRQoL). For longitudinal data analyses, generalised estimation equations, linear mixed effects modelling or cross-lagged panel models will be used. If needed, multiple imputation methods will be used to replace missing values.

Our study will also generate qualitative data as some questions require a descriptive response from participants. The analysis and reporting of these findings will follow the Consolidated criteria for Reporting Qualitative Research. $^{51}$

\section{Patient and public involvement}

In Finland, 15 young patients who participated in a transition camp prior to their transfer of care gave feedback on the first draft of the questionnaire. They were satisfied with the content of the questionnaire and especially highlighted the importance of including questions regarding their feelings, support needs and social and educational transitions in addition to the medical outcomes. Summaries of study results will be disseminated to study participants via email/mail.

\section{DISCUSSION}

The Bridge study is an international, prospective observational study that plans to follow adolescents across the critical years when their specialist medical care is transferred from child-oriented to adult-oriented health services. Patient follow-up is expected to be completed in 2022. By using a predetermined data collection and analysis plan, this study will fill several of the evidence gaps regarding transition of care.

An observational cohort design was chosen as the predictive value of long-standing transition recommendations remains understudied. The international study setting increases the generalisability and validity of the results. The study sites are in countries that have publicly funded healthcare systems to minimise the effect of private insurance and other financial barriers on adolescents' access to healthcare. To reduce selection bias, participants were consecutively recruited until the required number was reached. To reduce response bias, in Finland, research nurses/assistants outside of the usual care team contacted patients. The prospective design will help to reduce recall bias as most questions inquire about recent experiences or emotions. Observation bias is unlikely due to the planned length of follow-up (2 years).

\section{Limitations}

Differences between the two study sites pose a number of limitations. These relate to systemic differences between the healthcare systems, the health services available and the legal frameworks allowing access to patient data in Finland and Australia. Other differences relate to the individual administrative and data access mechanisms present across the two sites, the variability in patient cohorts (inclusion of those with intellectual disabilities, autism spectrum disorder and behavioural difficulties in Australia) and parental data availability after transfer (parent/guardian questionnaire data is only available from the Australian cohort and patients under 18 years in Finland). Attempts to accommodate these differences to allow comparison of the cohorts will be maximised.

Despite efforts to reduce bias, some patients may be lost to follow-up, a feature of other transition studies, which may skew the study results. This is likely to be a greater limitation for the Australian cohort as patients are transferred to multiple adult hospitals, each with differing administrative and medical record systems, in comparison to Finland where the majority are expected to transfer to a single adult service. In case of attrition $>20 \%$, we will conduct careful analyses between the baseline characteristics of retained and lost participants. ${ }^{52}$ Some selection bias may have occurred as Australian patients who were invited to participate by their transition care team may have been more likely to participate if they were receiving support from the transition support service. The impact on the outcomes are unclear, as while some patients will be referred to the transition team due to greater needs, they may also be expected to receive greater transition support.

The study population is patients who can independently respond to study questionnaires, due to the well-known discrepancies between patient and proxy responses. ${ }^{53} 54$ Patients whose education and employment are most severely affected by their chronic illness or disability, such as young people with intellectual disability or communication challenges, are therefore not included, which will reduce the representativeness of the study.

The COVID-19 pandemic will create unanticipated limitations. Infection control measures have led to major disruptions and sudden changes in the care of many groups of patients with chronic health conditions across both paediatric and adult systems. At both sites, this includes a rapid shift to telehealth instead of faceto-face consultations, and potential delays in the timing of transfer and first visit to adult services. Furthermore, the effect of COVID-19 may affect other aspects, such as emotional health and well-being, and wider impacts on engagement in education and employment. ${ }^{55}$ For this 
reason, we have decided to add questions regarding the effects of COVID-19 to the follow-up questionnaires.

\section{CONCLUSION}

Successful transition is often judged using data from specific disease measures such as laboratory values and medication adherence. Other measures of transition, including the psychosocial impacts of the process, are understudied and may benefit from greater attention. The Bridge study is designed to assess both of these aspects in an approach that aims to address holistic healthcare needs for adolescents transitioning to adult healthcare, and their families.

\section{Ethics and dissemination}

The Bridge study conforms to the Declaration of Helsinki. ${ }^{56}$ The Ethics Committee for Women's and Children's Health and Psychiatry at the Helsinki University Hospital (HUS/1547/2017) and the RCH Human Research Ethics Committee (38035) have approved the Bridge study protocol. Adolescents and their participating parents/guardians have received both verbal and written information explaining the purpose of the study and they have provided informed consent. Adolescents receive a minor compensation for filling the research questionnaires which take $30-45 \mathrm{~min}$ to complete. In Finland, patients aged 15 or older may provide consent to participate without approval from their parents.

Results will be presented at scientific meetings and published in international peer-reviewed journals. Summaries will be provided to the funders of the study as well as patients and their parents/guardians.

\footnotetext{
Author affiliations

${ }^{1}$ Pediatric Research Center, New Children's Hospital, Helsinki, Finland

${ }^{2}$ Helsinki University Hospital and University of Helsinki, Helsinki, Finland

${ }^{3}$ Transition Support Service, Royal Children's Hospital, Parkville, Victoria, Australia

${ }^{4}$ Health Services, Murdoch Children's Research Institute, Melbourne, Victoria,

Australia

${ }^{5}$ Nursing Science, University of Turku, Turku, Finland

${ }^{6}$ Clinical Research, University of Helsinki, Helsinki, Finland

${ }^{7}$ Department of Adolescent Medicine, Royal Children's Hospital, Parkville, Victoria, Australia

${ }^{8}$ Department of Paediatrics, The University of Melbourne, Parkville, Victoria, Australia

${ }^{9}$ Faculty of Medicine and Medical Technology, Tampere University, Tampere, Finland

${ }^{10}$ Stem Cells and Metabolism Research Program, Faculty of Medicine, University of Helsinki, Helsinki, Finland

${ }^{11}$ Centre for Adolescent Health, Murdoch Children's Research Institute and the Royal Children's Hospital, Parkville, Victoria, Australia
}

\section{Twitter Silja Kosola @siljakosola and Susan Sawyer @SusanSawyer01}

Acknowledgements The authors thank study participants and patient advisers, research nurse Satu Lindström for patient recruitment and biostatistician Pasi Aronen for checking both the power calculations and analysis plans.

Contributors The study concept and design was conceived by SK, EC and SS. HL, PJM, K-LK, TR, MT and KA assisted in refining the study questionnaires and study design. SK, EC, HL, AT and MK are responsible for data collection. Analyses will be conducted by SK, HL, AT and MK with the help of statisticians. SK prepared the first draft of the manuscript. All authors critically revised the manuscript and approved the submitted version.
Funding This study is supported by grants from the Foundation for Medical Research, the Helsinki University Hospital (TYH2019312), the Foundation for Pediatric Research, the Paulo Foundation and the Royal Children's Hospital Foundation (2018-991).

Competing interests None declared.

Patient and public involvement Patients and/or the public were involved in the design, or conduct, or reporting, or dissemination plans of this research. Refer to the Methods section for further details.

Patient consent for publication Not required.

Provenance and peer review Not commissioned; externally peer reviewed.

Open access This is an open access article distributed in accordance with the Creative Commons Attribution Non Commercial (CC BY-NC 4.0) license, which permits others to distribute, remix, adapt, build upon this work non-commercially, and license their derivative works on different terms, provided the original work is properly cited, appropriate credit is given, any changes made indicated, and the use is non-commercial. See: http://creativecommons.org/licenses/by-nc/4.0/.

\section{ORCID iD}

Silja Kosola http://orcid.org/0000-0002-2881-8299

\section{REFERENCES}

1 Raissadati A, Nieminen H, Jokinen E, et al. Progress in late results among pediatric cardiac surgery patients: a population-based 6-decade study with 98\% follow-up. Circulation 2015;131:347-53.

2 Lehtinen P, Ashorn M, Iltanen S, et al. Incidence trends of pediatric inflammatory bowel disease in Finland, 1987-2003, a nationwide study. Inflamm Bowel Dis 2011;17:1778-83.

3 Incidence Trends of Type 1 and Type 2 Diabetes among Youths, 2002-2012 | Enhanced Reader. chrome-extension:// dagcmkpagjlhakfdhnbomgmjdpkdklff/enhanced-reader.html? openApp\&pdf=https\%3A\%2F\%2F. Available: www.nejm.org\% 2Fdoi\%2Fpdf\%2F10.1056\%2FNEJMoa1610187\%3FarticleTools\% 3Dtrue [Accessed 22 Dec 2020].

4 Tuomilehto J, Ogle GD, Lund-Blix NA. Update on worldwide trends in occurrence of childhood type 1 diabetes in 2020. 17. Pediatric endocrinology reviews: PER, 2020.

5 Sawyer SM, Drew S, Yeo MS, et al. Adolescents with a chronic condition: challenges living, challenges treating. The Lancet 2007;369:1481-9.

6 Blum RW, Garell D, Hodgman CH. Transition from child-centered to adult health-care systems for adolescents with chronic conditions. A position paper of the Society for adolescent medicine. Journal of Adolescent Health 1993;14.

7 Bell LE, Bartosh SM, Davis CL. Adolescent transition to adult care in solid organ transplantation: a consensus conference report. In: American Journal of Transplantation, 2008: 2230-42.

8 Sable C, Foster E, Uzark K, et al. Best practices in managing transition to adulthood for adolescents with congenital heart disease: the transition process and medical and psychosocial issues: a scientific statement from the American heart association. Circulation 2011;123:1454-85.

9 Andrade DM, Bassett AS, Bercovici E, et al. Epilepsy: transition from pediatric to adult care. recommendations of the Ontario epilepsy implementation Task force. Epilepsia 2017;58:1502-17.

10 Peters A, Laffel L, Albright A. Diabetes care for emerging adults: recommendations for transition from pediatric to adult diabetes care systems: a position statement of the ADA, with representation by the ACOFP, the AAP, the AACE, the AOA the CDCP, children with Diabet., the ES, the ISPAD, JDRFI, the Natl. Diabet. Educ. Prog., and the Pediat. Endocr. soc. (formerly Lawson Wilkins Ped. Endocr. soc.). Diabetes Care 2011;34:2477-85.

11 Brooks AJ, Smith PJ, Cohen R, et al. Uk guideline on transition of adolescent and young persons with chronic digestive diseases from paediatric to adult care. Gut 2017;66:988-1000.

12 White PH, Cooley WC. Supporting the health care transition from adolescence to adulthood in the medical home from the American Academy of pediatrics, 2018. Available: www.aappublications.org/ news [Accessed 5 Apr 2021].

13 Willis ER, McDonagh JE. Transition from children's to adults' services for young people using health or social care services (NICE guideline NG43). Arch Dis Child Educ Pract Ed 2018;103:253-6.

14 Gray WN, Schaefer MR, Resmini-Rawlinson A, et al. Barriers to transition from pediatric to adult care: a systematic review. J Pediatr Psychol 2018;43:488-502. 
15 Crawford K, Wilson C, Low JK, et al. Transitioning adolescents to adult nephrology care: a systematic review of the experiences of adolescents, parents, and health professionals. Pediatr Nephrol 2020;35:555-67.

16 Hait EJ, Barendse RM, Arnold JH, et al. Transition of adolescents with inflammatory bowel disease from pediatric to adult care: a survey of adult Gastroenterologists. J Pediatr Gastroenterol Nutr 2009;48:61-5.

17 Peter NG, Forke CM, Ginsburg KR, et al. Transition from pediatric to adult care: internists' perspectives. Pediatrics 2009;123:417-23.

18 Michaud S, Dasgupta K, Bell L, et al. Adult care providers' perspectives on the transition to adult care for emerging adults with type 1 diabetes: a cross-sectional survey. Diabet Med 2018;35:846-54.

19 Nabbout $\mathrm{R}$, Teng $\mathrm{T}$, Chemaly $\mathrm{N}$, et al. Transition of patients with childhood onset epilepsy: perspectives from pediatric and adult neurologists. Epilepsy Behav 2020;104:106889.

20 Kosola S, Ylinen E, Finne P, et al. Implementation of a transition model to adult care may not be enough to improve results: national study of kidney transplant recipients. Clin Transplant 2019;33:e13449.

21 Annunziato RA, Emre S, Shneider B, et al. Adherence and medical outcomes in pediatric liver transplant recipients who transition to adult services. Pediatr Transplant 2007;11:608-14.

22 Prior M, McManus M, White P, et al. Measuring the "triple aim" in transition care: a systematic review. Pediatrics 2014;134:e1648-61.

23 Campbell F, Biggs K, Aldiss SK, et al. Transition of care for adolescents from paediatric services to adult health services. Cochrane Database Syst Rev 2016;3.

24 Schmidt A, llango SM, McManus MA, et al. Outcomes of pediatric to adult health care transition interventions: an updated systematic review. J Pediatr Nurs 2020;51:92-107.

25 Hart LC, Patel-Nguyen SV, Merkley MG, et al. An evidence map for interventions addressing transition from pediatric to adult care: a systematic review of systematic reviews. J Pediatr Nurs 2019;48:18-34.

26 Rutishauser C, Sawyer SM, Ambresin A-E. Transition of young people with chronic conditions: a cross-sectional study of patient perceptions before and after transfer from pediatric to adult health care. Eur J Pediatr 2014;173:1067-74.

27 Sawyer SM, McNeil R, Francis KL, et al. The age of paediatrics. Lancet Child Adolesc Health 2019;3:822-30.

28 Statistics Finland. Persons with the status of a child by age. Available: http://pxnet2.stat.fi/PXWeb/pxweb/en/StatFin/StatFin vrm perh/statfin perh_pxt 12c7.px/ [Accessed 5 Nov 2020].

29 Australian Institute of Family Studies. More young adults living at home with their parents | Australian Institute of family studies. Available: https://aifs.gov.au/media-releases/more-young-adultsliving-home-their-parents [Accessed 5 Nov 2020].

30 Finland, the fourth EU country where young people leave homes at earlier age. Available: https://www.foreigner.fi/articulo/lifestyle/ finland-the-third-eu-country-where-young-people-leave-beforehome/20190516174044001915.html [Accessed 5 Nov 2020].

31 Mikkonen J, Moustgaard H, Remes $\mathrm{H}$, et al. The population impact of childhood health conditions on dropout from Upper-Secondary education. J Pediatr 2018;196:283-90.

32 Sawyer SM, Ambresin A-E. Successful transitions: beyond disease control to better life chances. J Adolesc Health 2014;54:365-6.

33 Suris J-C, Akre C. Key elements for, and indicators of, a successful transition: an international Delphi study. Journal of Adolescent Health 2015;56:612-8.

34 Fair C, Cuttance J, Sharma N, et al. International and interdisciplinary identification of health care transition outcomes. JAMA Pediatr 2016;170:205

35 Gabriel P, McManus M, Rogers K, et al. Outcome evidence for structured pediatric to adult health care transition interventions: a systematic review. J Pediatr 2017;188:263-9.
36 Moynihan M, Saewyc E, Whitehouse S, et al. Assessing readiness for transition from paediatric to adult health care: revision and psychometric evaluation of the am I on TRAC for adult care questionnaire. J Adv Nurs 2015;71:1324-35.

37 Tsuda S, Kunisaki R, Kato J, et al. Patient self-reported symptoms using visual analog scales are useful to estimate endoscopic activity in ulcerative colitis. Intest Res 2018;16:579-87.

38 Rojkovich B, Gibson T. Day and night pain measurement in rheumatoid arthritis. Ann Rheum Dis 1998;57:434-6.

39 Backström M, Tynjälä P, Ylijoki H, et al. Finding specific 10-joint juvenile arthritis disease activity score (JADAS10) and clinical JADAS10 cut-off values for disease activity levels in nonsystemic juvenile idiopathic arthritis: a Finnish multicentre study. Rheumatology 2016;55:615-23.

40 DiMeglio LA, Acerini CL, Codner E, et al. ISPAD clinical practice consensus guidelines 2018: glycemic control targets and glucose monitoring for children, adolescents, and young adults with diabetes. Pediatr Diabetes 2018;19 Suppl 27:105-14.

41 Apajasalo M, Sintonen H, Holmberg C, et al. Quality of life in early adolescence: a sixteendimensional health-related measure (16D). Quality of Life Research 1996;5:205-11

42 Varni JW, Seid M, Kurtin PS. PedsQL 4.0: reliability and validity of the pediatric quality of life inventory version 4.0 generic core scales in healthy and patient populations. Med Care 2001;39:800-12

43 Spielberger CD. State-Trait anxiety inventory for adults 1983.

44 Marteau TM, Bekker $\mathrm{H}$. The development of a six-item short-form of the state scale of the Spielberger State-Trait anxiety inventory (STAl). Br J Clin Psychol 1992;31:301-6.

45 Kessler RC, Barker PR, Colpe LJ. Screening for serious mental illness in the general population.

46 Sawyer SM, Ambresin A-E, Bennett KE, et al. A measurement framework for quality health care for adolescents in hospital. $J$ Adolesc Health 2014:55:484-90.

47 Pirskanen M. Promoting adolescents' abstinence from substance use. Kuopio University Publications, thesis, 2007. Available: https:// epublications.uef.fi/pub/urn isbn 978-951-27-0510-8/urn isbn 978951-27-0510-8.pdf [Accessed 5 Nov 2020]

48 Goldenring J, Cohen E. Getting into adolescent heads. contemporary pediatrics, 1988. Available: https://www.contempo rarypediatrics.com/view/getting-adolescent-heads [Accessed 5 Nov 2020].

49 White $\mathrm{PH}$, Cooley WC. Supporting the health care transition from adolescence to adulthood in the medical home from the American Academy of pediatrics guidance for the clinician in rendering pediatric care, 2018. Available: www.aappublications.org/news

50 von Elm E, Altman DG, Egger M, et al. The strengthening the reporting of observational studies in epidemiology (STROBE) statement: guidelines for reporting observational studies. J Clin Epidemiol 2008;61:344-9.

51 Tong A, Sainsbury P, Craig J. Consolidated criteria for reporting qualitative research (COREQ): a 32-item checklist for interviews and focus groups. Int J Qual Health Care 2007;19:349-57.

52 Bankhead C, Aronson JK, Catalogue of Bias Collaboration, Attrition bias - Catalogue of Bias. Catalogue of Bias 2017 https:// catalogofbias.org/biases/attrition-bias/

53 Eiser C, Jenney M. Measuring quality of life. Arch Dis Child 2007:92:348-50.

54 Kosola S, Lampela H, Lauronen J, et al. General health, healthrelated quality of life and sexual health after pediatric liver transplantation: a nationwide study. Am J Transplant 2012;12:420-7.

55 Jansen D, Kosola S, Arevalo LC, et al. Child and adolescent health needs attention now, and in the aftermath of the COVID-19 pandemic. Int J Public Health 2020;65:723-5.

56 World Medical Association. World Medical association Declaration of Helsinki. Ethical principles for medical research involving human subjects 2013 